\title{
Case report: aortic bioprosthesis marantic endocarditis
}

\section{Clara Douin , Stella Marchetta , Raluca Dulgheru, Pierre-Julien Bruyères , Marie Moonen \& Patrizio Lancellotti}

To cite this article: Clara Douin , Stella Marchetta , Raluca Dulgheru , Pierre-Julien Bruyères , Marie Moonen \& Patrizio Lancellotti (2020): Case report: aortic bioprosthesis marantic endocarditis, Acta Cardiologica, DOI: 10.1080/00015385.2020.1813995

To link to this article: https://doi.org/10.1080/00015385.2020.1813995

\section{册Published online: 13 Oct 2020.}

Submit your article to this journal $₫$

Q View related articles $\sqsubset$

View Crossmark data 


\title{
Case report: aortic bioprosthesis marantic endocarditis
}

\author{
Clara Douin $^{a}$, Stella Marchetta ${ }^{b}$, Raluca Dulgheru ${ }^{a}$, Pierre-Julien Bruyères ${ }^{a}$, Marie Moonen ${ }^{a}$ and \\ Patrizio Lancellotti ${ }^{\mathrm{a}, \mathrm{c}}$ \\ ${ }^{a}$ GIGA Cardiovascular Sciences, Department of Cardiology and Radiology, University of Liège Hospital, CHU Sart-Tilman, Liège, \\ Belgium; 'bepartment of Cardiology, CHC MontLegia Hospital, Liège, Belgium; 'Gruppo Villa Maria Care and Research, Maria Cecilia \\ Hospital, Cotignola, and Anthea Hospital, Bari, Italy
}

ARTICLE HISTORY Received 13 May 2020; Revised 17 August 2020; Accepted 18 August 2020

KEYWORDS: Endocarditis; melanoma; Edoxaban; vascular disease

We describe the case of a 75-year-old man presenting to the emergency with a stage III dyspnoea evolving for several months. His main medical history was an aortic valve replacement 8 years ago, a paroxysmal atrial fibrillation anticoagulated by Edoxaban $60 \mathrm{mg}$ daily and a metastatic melanoma in progression despite ongoing immunotherapy. In front of this dyspnoea, the oncologist suspected a toxic pneumopathy due to Pembrolizumab requiring the interruption of this medication and administration of systemic corticotherapy. In the absence of clinical improvement, a transthoracic cardiac ultrasound was performed and reported a bioprosthetic stenosis: peak aortic velocity of $4.73 \mathrm{~m} / \mathrm{s}$, peak and mean gradients of $89 \mathrm{mmHg}$ and $50 \mathrm{mmHg}$ and a calculated valvular area of $0.9 \mathrm{~cm}^{2}$ according to the continuity equation (Panel A). The transesophageal echocardiography revealed a diffuse thickening of the aortic leaflets (Panel B). A cardiac multi-detector computed tomography was realised and identified a thrombus between the right and the non-coronary leaflets (Panel C). An infective endocarditis then was excluded: negative blood cultures, negative inflammatory syndrome, absence of fixation at ${ }^{18}$ FDG PET-CT. Edoxaban was replaced by Enoxaparin with rapid improvement in symptoms and reopening of the valve at the control of echocardiography (Panel D).

We have known for long that cancers can be associated with a hypercoagulabilty state and that high doses of corticotherapy can also exacerbate it. In the literature, there is no other reported case of bioprosthetic thrombosis in metastasised melanomas. This case also illustrated that the use of direct oral anticoagulant (DOACs) was unable to prevent valve thrombosis in the present oncologic context.

\section{Disclosure statement}

No potential conflict of interest was reported by the author(s).

CONTACT Patrizio Lancellotti plancellotti@chuliege.be $=$ Department of Cardiology, University of Liège Hospital, Domaine Universitaire du Sart Tilman, B.35-4000, Liège, Belgium

(C) 2020 Belgian Society of Cardiology 
Panel A

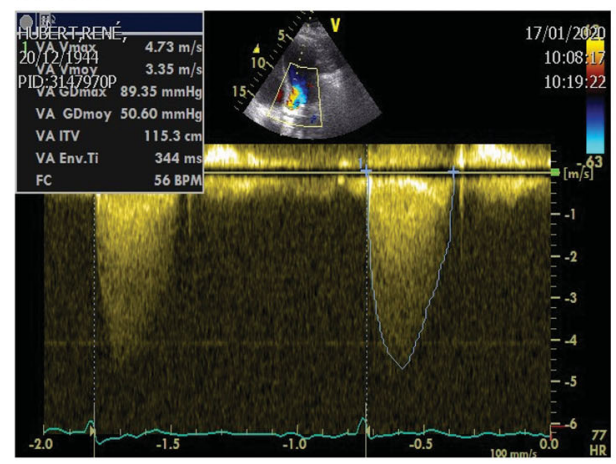

Panel B

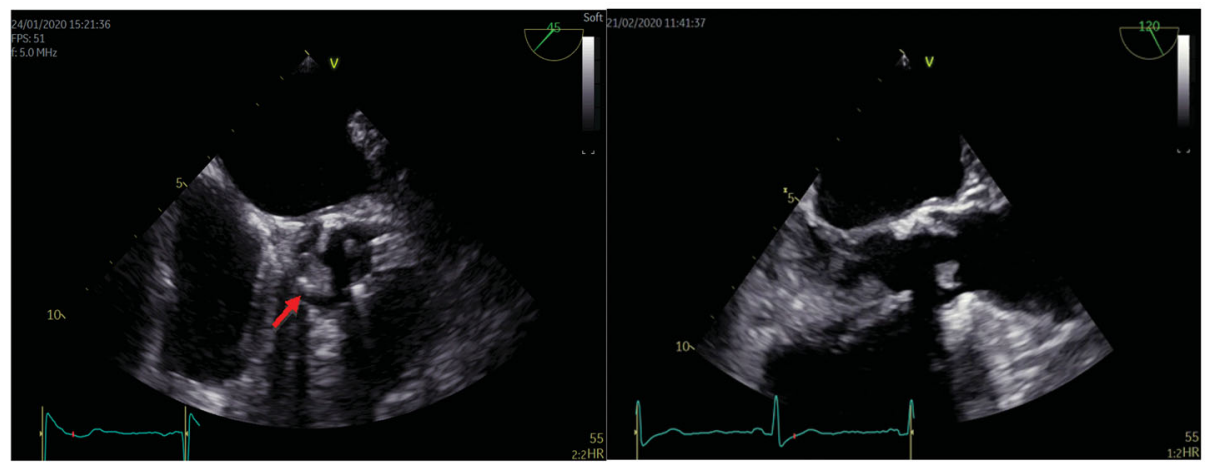

Panel C

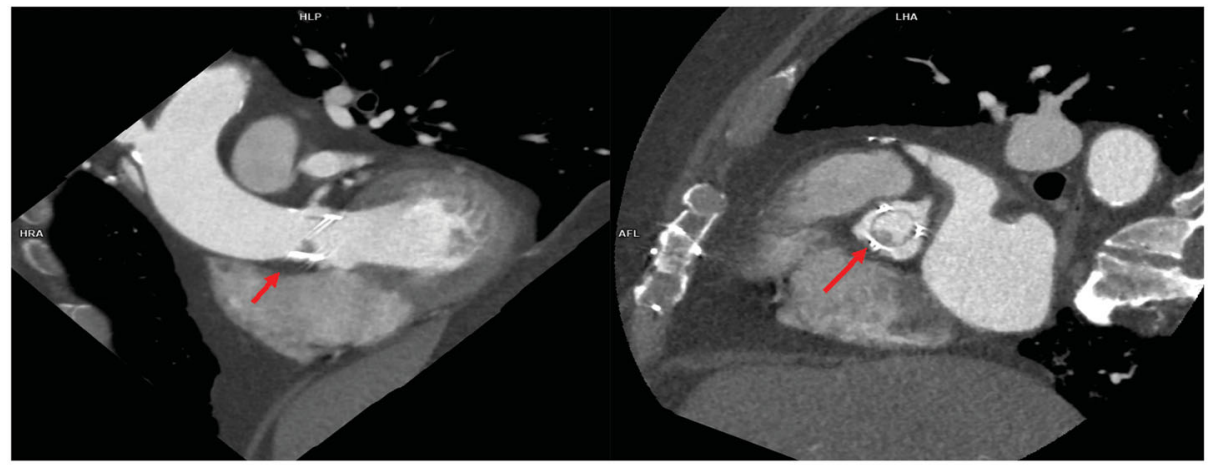

Panel D

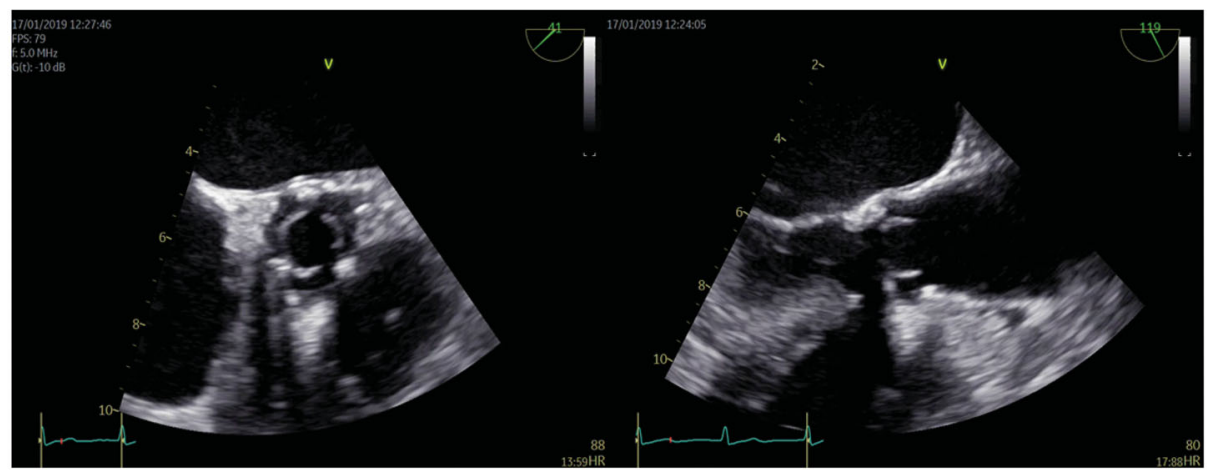

Panel A: Increased trans aortic pressure gradients and peak velocity revealed by a transthoracic echocardiography. Panel B: Diffuse thickening and reduced mobility of the aortic leaflets showed by transesophageal echocardiography. Panel C: Thrombus (hypo attenuating opacity during systole and diastole) between the right and the non-coronary cups pointed by the arrow on these cardiac multi-computed tomography images. Panel D: Second transesophageal echocardiography after Enoxaparin therapy: improvement of the aortic leaflets thickening and much better opening of the bioprosthetic valve. 